\title{
Diagnóstico dos problemas que interferem nas ações do PSF do município de Araruama: sugerindo o empowerment como teoria organizacional
}

\author{
Diagnosis of the problems interfering with the actions of the \\ Family Health Program in the city of Araruama: suggesting \\ empowerment as an organizational theory
}

Simone Grativol Marchon ${ }^{1}$ Zeilma da Cunha ${ }^{2}$

\section{Resumo}

$\mathrm{Na}$ atual conjuntura política de saúde brasileira, o PSF tem desempenhado papel importante para a construção e consolidação do Sistema Único de Saúde (SUS). Considerando esta concepção, observa-se que as políticas de saúde se materializam nos serviços, mediante as ações de seus atores sociais e suas práticas cotidianas, buscando refletir sobre as práticas diárias em saúde, no contexto do Programa Saúde da Família (PSF). Este estudo buscou identificar as principais barreiras sociodemográficas, infra-estrutura e comportamentais, apontadas pelos profissionais de saúde do PSF do município de Araruama. De acordo com os resultados, foi sugerido o empowerment como teoria organizacional de gestão. O conceito de empowerment utilizado sugere a mudança da mentalidade tradicional de comando e controle para a criação de um ambiente apoiador, onde as pessoas possam crescer e dar o melhor de si, em benefício da organização. Estudo epidemiológico descritivo, com aplicação de entrevista semi-estruturada para obter informações sobre as principais barreiras no desempenho das funções e conhecer as possíveis associações existentes entre estes fatores. Participaram do estudo 82 profissionais de saúde das oito equipes do PSF Araruama. Os resultados dos fatores sociodemográficos indicaram que 50\% atuam no programa por mais de cinco anos e relataram desgaste na atividade por diversos motivos: longa permanência no mesmo local de trabalho; repetição das mesmas vivências; relações conflituosas entre os profissionais da equipe; falta de motivação; falta de resolubilidade dos problemas da comunidade e renda mensal insatisfatória. Quanto aos resultados relacionados à infra-estrutura, 77\% consideraram suas atividades profissionais desgastantes devido às condições de trabalho. Os fatores comportamentais indicaram como principal barreira à relação entre as equipes, entre estes, a falta de consenso entre os profissionais, as divergências e o poder do médico ou enfermeiro, reduzindo o comprometimento e o diálogo entre eles e a comunidade. O empowerment estimula o mecanismo de escuta e interação permanente entre gestores e profissionais de saúde e a comunidade, de forma que suas opiniões e percepções sejam valorizadas nos processos de mudança. A falta de estímulo, conflitos e falta de comprometimento entre os membros da equipe podem ser minimizados pela utilização das dimensões individuais, organizacionais e comunitárias do empowerment, criando novas estratégias de ações por meio do processo contínuo de relacionamento entre os membros da equipe.

Palavras-chave: Saúde da Família; Sistema Único de Saúde; Gestão em Saúde; Empoderamento.
Key Words: Family Health; Brazilian Health

System; Health Management; Empowerment.

${ }^{1}$ Mestre em Saúde da Família, Programa de Pós-Graduação em Saúde da Família, Universidade Estácio de Sá, Rio de Janeiro, Brasil.

${ }^{2}$ Doctor in Education, Columbia University, New York, N.Y, Programa de Pós-Graduação em Saúde da Família, Universidade Estácio de Sá, Rio de Janeiro, Brasil. 


\section{Abstract}

In the current Brazilian bealth policy, the Family Health Program (FHP) is playing an important role in the construction and consolidation of the Brazilian Health System. Within this conception it can be observed that the health policies are put in practice in the services, through the actions and daily practices of the social actors in the context of the FHP. The purpose of this study was to identify the main barriers related to infra-structure, behavior and social-demographic factors pointed out by the health professionals working in the FHP in the city of Araruama. According to the results, empowerment was suggested as an organizational theory. The concept of empowerment used refers to changing the traditional mentality of command and control, creating a supportive environment allowing for people to develop a new mentality and give their best for the benefit of the organization. This epidemiological descriptive study used semi-structured interviews to collect information about the major barriers for the performance of the professionals and about possible associations between those factors. Eighty-two health professionals from the eight FHP teams of Araruama participated in this study. The results of socio-economic factors indicated that $50 \%$ participate in the program for more than five years and dislike their activity for the following reasons: long permanence at the same work place; repetition of the same experiences; conflicts among team members; lack of motivation; lack of solutions for the problems of the community and low wages. In relation to infrastructure, the results demonstrated that $77 \%$ consider their activities distressing due to the poor working conditions. The behavioral factors indicated as the major barrier the relationship of the team members, in special the lack of consensus among the professionals, disagreements, and the power of doctors and nurses reducing their commitment and the dialogue among them and with the community. The empowerment stimulates the mechanisms for listening and promotes interaction among managers, health professionals and the community so that their opinions and suggestions can be taken into consideration in the process of change. The lack of motivation, conflicts and lack of commitment among the team members can be minimized using the individual, organizational and community dimensions of empowerment creating new strategies through continued relationship among the team members.

\section{Introdução}

O Pacto Pela Saúde 2006 busca a consolidação do Sistema Único de Saúde (SUS), tendo como um dos seus objetivos, o fortalecimento da Atenção Básica à Saúde, sendo a Estratégia Saúde da Família (ESF) considerado como modelo e centro ordenador das redes de atenção à saúde do SUS ${ }^{1}$. Neste cenário, a ESF se fortaleceu ampliando suas possibilidades de intervenção na promoção da saúde, devido às ações educativas, preventivas e de mobilização social, passando a fazer parte dos programas de Saúde Coletiva.

Embora alcançados inúmeros avanços demonstrados por meio dos dados epidemiológicos, as unidades de SF convivem de modo contrário aos problemas diversos, tais como o baixo investimento na qualificação dos trabalhadores, especialmente no que se refere à gestão participativa e ao trabalho em equipe multiprofissional. Ainda, os baixos salários, indefinição no vínculo contratual dos funcionários e a disparidade salarial são fatores que ainda interferem na atuação do trabalho em equipe, desestimulando a coparticipação do gestor/funcionário, desvalorizando e excluindo os trabalhadores e usuários no processo de promoção da saúde.

Os resultados apresentados na pesquisa realizada por Machado sobre o perfil dos profissionais médicos e enfermeiros do PSF no Brasil demonstraram que a maioria dos profissionais considera ser desgastante o exercício de suas atividades na SF. Foram consideradas como as principais causas de desgaste no exercício da profissão: o vínculo trabalhista precário; o excesso de trabalho; a falta de recursos humanos capacitados, materiais e medicamentos; a dificuldade de acesso às áreas de trabalho; a baixa remuneração e a falha no sistema de referência e contra-referência ${ }^{2}$.

Segundo documento publicado pelo Ministério da Saúde, foi verificado alguns pontos que precisam ser observados com atenção pelos gestores, tais como: rede de referência e contra-referência; a comunicação intrasetorial e intersetorial (integração dos setores); o envolvimento comunitário e controle social, os quais trazem, como conseqüência, o distanciamento dos profissionais pela falta de 
compromisso, responsabilidade e desmotivação, causando a baixa resolubilidade no atendimento médico ${ }^{3}$.

Considerando a complexidade dos fatores envolvidos no sistema de saúde em geral e mais especificamente na ESF, é importante que haja uma reformulação nas estratégias, contribuindo para a efetividade das ações. Neste contexto, o presente trabalho buscou diagnosticar as principais barreiras relacionadas aos fatores sociodemográficos, de infra-estrutura e comportamentais apontadas pela equipe dos profissionais de saúde do Programa Saúde da Família no Município de Araruama, sugerindo a utilização do empowerment como teoria organizacional.

O conceito de empowerment é utilizado neste estudo como "a expansão de vantagens e capacidades (ou habilidades) de pessoas para participar, negociar, influenciar, controlar e sustentar incontáveis instituições que afetam suas vidas em busca do bem comum"4.

\section{Métodos}

O município de Araruama, localizado no estado do Rio de Janeiro, baixada litorânea, com área de 635,40 $\mathrm{Km}^{2}$ e população estimada de 110 mil habitantes (IBGE, 2007).

Nesta pesquisa, foi utilizado um estudo epidemiológico descritivo preliminar, por meio da aplicação de inquérito transversal, semi-estruturado e previamente validado. Participaram do estudo todos os profissionais de saúde médicos, enfermeiros, técnicos de enfermagem e agentes comunitários de saúde - das oito unidades integrantes das equipes da SF do município de Araruama, perfazendo um total de 82 profissionais.

O estudo foi conduzido em duas etapas, sendo a primeira o diagnóstico das percepções e práticas dos profissionais de saúde que atuam na Saúde da Família no município de Araruama, por meio do questionário semiestruturado, em que foram observados os fatores sociodemográficos, de infra-estrutura e comportamentais. Com a utilização do software epiinfo 6.04, foram analisados os dados da primeira etapa, e, com base nos resultados, foram sugeridas, na segunda etapa, estratégias para solução das bar- reiras encontradas, sugerindo como ferramenta a utilização do empowerment como teoria organizacional, individual e comunitária.

A presente pesquisa foi realizada mediante a avaliação e autorização do Comitê de Ética em Pesquisa da Universidade Estácio de Sá e autorização dos profissionais das unidades do Programa Saúde da Família do município de Araruama.

\section{Resultados e Discussões}

Para o levantamento das barreiras associadas aos fatores sociodemográficos, foram analisados os itens relacionados à renda, ao grau de instrução, ao tempo de formação e ao período do tempo de trabalho em unidades do PSF. Dentre as principais barreiras que interferem na produtividade e resolubilidade dos problemas, 91\% dos profissionais de saúde consideraram a atividade profissional diária desgastante. Os motivos citados pelos profissionais foram: a renda mensal insatisfatória, extensa carga horária acrescida de um número excessivo de famílias cadastradas, outros vínculos empregatícios, para melhorar a renda mensal. Ainda, o vínculo empregatício foi considerado como a principal barreira por $79 \%$ dos profissionais, pela diversidade de contrato de trabalho, não garantindo uma política de recursos humanos, gerando descontentamento dos profissionais.

Entre os agentes comunitários de saúde, 83\% encontram-se na faixa correspondente a baixo nível de escolaridade, e 55\% dos outros profissionais das equipes apresentaram baixa capacitação para o exercício de suas funções. Portanto, a falta de profissionais com "perfil" para atividades de saúde gera o baixo comprometimento com a comunidade e os problemas de saúde pública, ocasionando conflitos de informações nas equipes e desmotivação profissional.

O empowerment individual refere-se à habilidade do indivíduo para tomar decisões e ter controle sobre sua própria vida ${ }^{5}$. Sendo assim, desmotivação dos profissionais, conflitos nas equipes e o baixo comprometimento profissional acima mencionado estão diretamente relacionados a 
este conceito de empowerment, em que o sentimento de maior controle sobre a própria vida, que os indivíduos experimentam, por pertencerem a distintos grupos, pode ocorrer sempre que haja necessidade de as pessoas participarem de ações coletivas, buscando a autonomia individual e coletiva.

Este nível do empowerment deve estar relacionado aos vários programas e gestão de recursos humanos, dentre eles, desenho de cargos, treinamento e desenvolvimento, remuneração e avaliação de desempenho ${ }^{6}$. Observando a necessidade de capacitação profissional, na qual resulta em desvalorização, desmotivação profissional e baixa estima do indivíduo, a concepção de empowerment individual poderá ser utilizada por meio da implementação de práticas educativas, que sempre incidem sobre a distribuição de poder na equipe, constituindo-se em mecanismo de valorização profissional.

Quanto aos fatores relacionados à infra-estrutura, foram verificadas como barreiras: localização predial, estrutura física e a falta de recursos para manutenção do mesmo; veículos para transporte e apoio; disponibilidade de remédios, insumos e bens patrimoniais. Noventa e cinco por cento dos profissionais indicaram a falta de recursos financeiros para a saúde como principal barreira, sem priorização para as unidades do PSF, contribuindo para a baixa resolubilidade dos procedimentos técnicos, e também a heterogeneidade na estrutura física das unidades do PSF's, consideradas inadequadas e em situação precária. Essas barreiras causam vários conflitos entre os profissionais e a comunidade, os quais buscam a unidade e não conseguem o atendimento desejado, por inadequação da estrutura física e relação conflituosa entre os profissionais e os gestores.

O empowerment organizacional sugere o envolvimento cooperativo de tomada de decisões que, juntos, somam esforços em direção à mesma meta, definida por todos $^{6}$. Este processo facilita o estreitamento das relações entre gestão e equipes de saúde, pela criação de grupos focais, onde sejam direcionados, conjuntamente, esforços para melhor utilização dos recursos financeiros nas unidades de SF. Desta forma, o empowerment organizacional deverá transformar-se em estratégia política, que se contrapõe às propostas de gestão e regulação de instituições que sustentam projetos verticalizados, geralmente iníquas e injustas, que não contribuem para a manutenção da ordem institucional.

Ainda, este tipo de empowerment irá beneficiar o plano institucional, atuando nos níveis crescentes de horizontalização das decisões, promovendo maior participação de todos os componentes da organização, na identificação e resolução de problemas que os afetam e no acesso à informação. Tais fatores são de grande importância para a avaliação do grau de empowerment organizacional quando se considera o aumento de resolubilidade e melhoraria no desempenho da instituição.

Neste estudo, foram também observadas as barreiras relacionadas aos fatores comportamentais, em que foram considerados: relacionamento entre os membros da equipe; relacionamento profissional/usuário; autonomia; competência e prestígio social. Os resultados indicaram que $81 \%$ dos profissionais entrevistados consideraram o poder institucional do profissional médico, reconhecidamente muito forte, perante os outros profissionais da equipe e perante a comunidade, como sendo outra barreira importante no relacionamento entre os membros das equipes. Portanto, justifica-se uma vez mais a aplicação da teoria do empowerment, que trabalha com a redistribuição de poder e a valorização do saber popular e dos outros profissionais. A dificuldade de comunicação existente entre o técnico, aquele que detém o saber legitimado pela ciência, e o usuário dos serviços públicos de saúde, oriundo das classes populares, interfere diretamente no processo de relacionamento entre as pessoas.

Segundo Valla ${ }^{7}$, o conhecimento das classes populares é o tempo todo desqualificado e infantilizado pelos profissionais de saúde. Porém, o autor afirma que estas classes conservam um saber próprio, tão importante quanto o dos técnicos, que vem da vivência do sofrimento e da falta de recursos que precisam ser considerados. Quando o usuário chega ao serviço de saúde, há uma história anterior, de vivências e experiências, que raramente é observada. 
Quase sempre, os recursos utilizados por essas classes são desmerecidos e considerados como "o erro". Nesse sentido, o próprio vocabulário diferenciado é utilizado, muitas vezes, como barreira entre o técnico e o usuário do serviço de saúde.

O conceito de empowerment comunitário sugere a elaboração de estratégias que promovam: a participação, visando ao aumento do controle sobre a vida por parte de indivíduos e comunidades; a eficácia política com maior justiça social e melhoria da qualidade de vida ${ }^{6}$.

É importante ressaltar que os conflitos presentes na relação das equipes, apontados neste estudo, quase sempre estão relacionados à falta de diálogo e consenso entre os próprios profissionais e entre gestores e profissionais. Exemplo da falta do vínculo entre a comunidade e as unidades do PSF, pela falta do diálogo, foram ressaltados nos depoimentos dos profissionais de saúde, demonstrando a redução no poder de fala ou limitações das suas ações ao relatarem o seguinte:

“(...) quase não conversamos entre nós, apenas nas reuniões de serviço, não dá tempo e o chefe não quer escutar" (ESF IGUABINHA). “(...) estou cansada de tentar falar sobre os meus problemas na reunião, mas não consigo falar, só a enfermeira fala" (ESF SÃO VICENTE).

O empowerment comunitário facilita o desenvolvimento do poder, por meio da união entre os membros da equipe, promovendo um esforço conjunto para o bem comum, com falas e decisões coletivas. A valorização do relato oral dos membros das equipes do PSF deve ser considerada como técnica importante para coleta de informação e avaliação do nível de relacionamento da equipe.

Neste tipo de empowerment, há uma forma diferente de intervir e de promover saúde na comunidade, ou seja, onde não se acredita que transformações ocorram, se partirem de um processo desigual, no qual os problemas são nomeados por outros que são estranhos às reais questões de determinada comunidade ou da equipe de saúde. Tais procedimentos ocorrem geralmente com o poder dos gestores de saúde, que impõem, verticalmente, soluções para os problemas, sem os conhecer, não abrindo espaço para o diálogo conjunto com os profissionais.

Em outras situações, a comunidade não reconheceu a SF como uma unidade de apoio, pois não consegue dar resolubilidade as necessidades de saúde dos usuários atendidos no local. Nessa relação, autores sugerem propostas de humanização das práticas médicas, nas quais se propõem vínculos mais efetivos e afetivos entre usuário e o profissional de saúde, levando-se em conta os fatores subjetivos $^{8}$.

Finalmente, espera-se que, por meio do processo de desenvolvimento dos diferentes níveis de empowerment organizacional, individual e comunitário, obtenha-se como resultado o aumento da capacidade dos indivíduos e coletividades, capazes de definirem, analisarem e atuarem sobre seus próprios problemas.

\section{Conclusão}

A teoria do empowerment tem sido amplamente utilizada na avaliação de grupos, indivíduos e comunidades, dentro do contexto social, político e institucional em que se inserem. No campo da saúde coletiva, por exemplo, acredita-se que um avanço substantivo na qualidade dos serviços e das ações de saúde somente é possível a partir do envolvimento efetivo da população, capaz de identificar os problemas e buscar soluções, garantindo, junto ao Estado, os direitos de integralidade e de qualidade dos serviços e dispositivos de saúde. Nesse sentido, profissionais de saúde e os usuários dos serviços de saúde têm um papel importante na garantia da qualidade dos serviços prestados pelas instituições de saúde, com a participação nos Conselhos de Saúde, garantida pela Lei no 8142/90?.

Embora o objetivo deste estudo não tenha sido a aplicação do empowerment, observamos nos relatos dos profissionais a necessidade urgente de aplicação de uma ferramenta capaz de modificar a atual realidade das unidades, representada pela grande desmotivação expressa nos resultados. Acreditamos ainda que, mais do que repassar informações e induzir determinados comportamentos, o empowerment busca apoiar pessoas e coletividades a reali- 
zarem suas próprias análises, para que tomem decisões consideradas corretas, desenvolvendo a consciência crítica e a capacidade de intervenção sobre a sua realidade. Dessa forma, o empowerment contribuirá para que os profissionais deixem o quadro de passividade, desmotivação e baixa mobilização, para uma posição ativa, criadora de novos espaços dentro da instituição, e os resultados do processo de empowerment vão se mostrar na relação equipe-equipe, equipe-profissional e equipe-comunidade.

As organizações que utilizam o empowerment são conduzidas democraticamente, e cada membro divide informação e poder, com participação ativa nos processos de tomada de decisão ${ }^{10}$. Para isso, todos os membros ou participantes devem ser envolvidos no planejamento, implementação, avaliação e controle de esforços, na direção de objetivos e planos mutuamente definidos. Conseqüentemente, se confere o empowerment aos indivíduos, como parte do processo organizacional, fazendo com que eles se sintam os "verdadeiros donos" neste processo de construção.

Acima de tudo, é importante destacar que o empowerment é melhor concebido e avaliado como processo contínuo, no qual os participantes passam do nível de fortalecimento pessoal para o desenvolvimento de pequenos grupos de apoio, de organizações comunitárias, parcerias e, finalmente, para a construção e organização de ação política.

\section{Referências}

1. Brasil. Ministério de Saúde. Portaria GM nº 648, de 28 de março de 2006. Aprova a Política Nacional de Atenção Básica, estabelece a revisão de diretrizes e normas para a organização da atenção Básica para Programa Saúde da Família e Programa de Agente Comunitário. Disponível em: <http:/ /www.saúde. gov.br/psf/programa.index.asp> . Acesso em: 06 agosto $2006 \mathrm{~b}$.

2. Machado MH. Perfil dos Médicos e Enfermeiros do Programa Saúde da Família no Brasil. Brasília (DF): Ministério da Saúde; 2000. v.1-8.

3. Brasil. Ministério de Saúde. Saúde da Família: estratégia para ampliação do modelo. Brasília (DF): Ministério da Saúde; DAB. 2002.

4. BIRD, Banco Mundial. Relatório sobre o desenvolvimento mundial 1993: investindo em saúde. Rio de Janeiro: Fundação Getúlio Vargas; 1993.

5. Wallerstein N, Bernstein E. Empowerment education: Freire's ideas adapted to health education. Health Educ Quaterly. 1988; 15:379-94.

6. Santos FCA. Dimensões competitivas e estratégias de recursos humanos. Gest. Prod. 1998; 8(3).

7. Valla V. A crise da compreensão é nossa: procurando compreender a fala das classes populares. Educação e Realidade. 1997; 22(2).

8. Caprara A, Franco ALS. A Relação paciente-médico: para uma humanização da prática médica. Cad. Saúde Pública. jul-set. 1999; 15(3):647-654.

9. Brasil. Ministério da Saúde. Lei 8142, de 28 de Dezembro de 1990. Diário Oficial da Republica Federativa do Brasil, Poder Executivo, Brasília, DF, dez. 1990b.

10. Cunha Z. et al. A Case-Study of a Curriculum Development Process in Nutrition Education Using Empowerment as Organational Policy. Ecology of Food and Nutrition. dez. 2000; 39: 417-435.

\section{Endereço para correspondência:}

Rua Senador Pompeu no 208, Bairro Parque Hotel - Araruama / RJ

CEP: 28970-000

\section{Endereço eletrônico:}

simonemarchon@oi.com.br 